\title{
XLV. On the theory of the dispersion of light
}

\section{S. Earnshaw M.A.}

To cite this article: S. Earnshaw M.A. (1842) XLV. On the theory of the dispersion of light, Philosophical Magazine Series 3, 20:131, 304-310, DOI: 10.1080/14786444208650573

To link to this article: http://dx.doi.org/10.1080/14786444208650573

册 Published online: 01 Jun 2009.

Submit your article to this journal 준

Џll Article views: 2

Q View related articles $₫$ 
304 Mr. Earnshaw on the Theory of the Dispersion of Light.

même de doter la science d'une pile construite d'après les principes précédentes, et qui est aujourd'hui généralement adoptée dans les expériences de physique."

It is only necessary to recall the data of my first paper upon the subject in the Philosophical Transactions, viz. February 11 th, 1836, and that of Prof. Grove's communication to the French Academy, I believe, April 1839. Prof. Grove has never spoken of his battery but as the further application of principles which I had previously deduced.

In conclusion, I cannot but express my regret that the filial piety of M. Edmond Becquerel should have betrayed him into an act of injustice by preferring a claim for his father which is totally unsupported by the facts of the case, and from which his well-earned reputation can derive no permanent extension. I remain, dear Sir, Very faithfully yours,

King's College, London, J. F. Daniell. March 1842.

To Richard Phillips, Esq., \&c. \&c.

XLV. On the Theory of the Dispersion of Light. By S. EARNshaw, M.A., Cambringe.

To the Editors of the Philosophical Magazine and Journal. Gentremen,

I HAVE observed with great interest the efforts which mathematicians have made to establish the undulatory theory of light on a firm basis. That theory (its fundamental hypothesis being admitted) has succeeded so well in accounting for a great variety of intricate and delicate phænomena, that I am not much surprised to observe that some of its leading admirers have adopted the opinion that its truth is now beyond controversy. Among its greatest advocates, however, are some who do not hesitate to allow that certain optical phænomena have not been fully accounted for on their favourite theory, and that others have been as yet explained only in a doubtfil manner. I hope therefore that, without incurring the risk of being reckoned an anti-undulationist, I may be permitted to make a few remarks on that "opprobrium of all theories-the dispersion of light." A compendious view of the present state of this portion of the general theory of undulations may be found in a book lately published by Professor Powell $*$. In that work are contained several sets of tables, which exhibit,

* The Undulatory Theory as applied to the Dispersion of Light, \&c. 
for a very extensive range of substances, the indices of refraction as found both by experiment and by theory. The near agreement of results, as there exhibited, is such as cannot fail to impress the reader, who is content with a general view of them, with most favourable sentiments respecting the theory from which they are derived. I have however dipped deeper into the matter, and by executing the calculations of several cases have arrived at results which I desire now to set before your readers, chiefly with a view of eliciting from some one such an explanation as shall induce me to assent to the opinion of Professor Powell, that "the theory of undulations supplies at once both the laws and the explanation of the phænomena of dispersion."

The first method of calculation is the one which was made use of by Professor Powell in three papers printed in the Transactions of the Royal Society, under the title "Researches towards establishing a theory of the Dispersion of Light," and distinguished as Nos. I. II. III. The formula employed is equivalent to the following:-

$$
\mu=H\left\{\frac{\left(\frac{A}{\lambda}\right)}{\sin \left(\frac{A}{\lambda}\right)}\right\} . \ldots \ldots
$$

This equation being directly furnished by theory, it is a matter of intense interest to see how far its truth is supported by experiment. I have not however met with any calculations founded upor it, except in the papers just referred to, and therefore it is that in my remarks upon it $I$ shall be under the necessity of referring to an inadvertence in those papers which I should otherwise gladly have left unmentioned. After comparing the results of theory with those furnished by experiment, Professor Powell comes to the conclusion that "the refractive indices are related to the lengths of waves, as nearly as possible, according to the formula deduced from $M$. Cauchy's theory." Now, if I mistake not, in all those papers, the author, by forgetting to reduce Fraunhofer's values of $\lambda$ in air to the proper medium, did in fact employ a formula which differs from the correct one furnished by theory; for, rectifying the oversight, the true formula supplied by M. Cauchy's theory is the following:-

$$
\mu=H \cdot \frac{\sin ^{-1}\left(\frac{A}{\lambda}\right)}{\left(\frac{A}{\lambda}\right)}, \ldots \ldots .
$$


306 Mr. Earnshaw on the Theory of the Dispersion of Light.

$H$ and $A$ being constant for the same medium, and the values of $\lambda$ to be used being those which belong to air.

Both these formulæ are of the form $\frac{\theta}{\sin \theta}$, but though from this circumstance they might be supposed to be similar, they do in fact materially differ; for in the former it is the arc $\theta$ which for different rays is proportional to $\frac{1}{\lambda}$, while in the latter it is the $\sin \theta$ which is proportional to $\frac{1}{\lambda} ;$ a distinction which will be considered very important, when it is remembered that the results are expected to be accurate in the third place of decimals, and to approximate to the figure in the fourth place, and that the values of $\theta$ are by no means small. Let us consider the case of flint glass, No. 13: the values of $\theta$ in "Research No. I." range from $16^{\circ} 10^{\prime}$ for letter B, to $27^{\circ} 39^{\prime}$ for $\mathrm{H}$. In the formula (2.) I find the corresponding values to be $15^{\circ} 21 \frac{1}{2}^{\prime}$ and $27^{\circ} 22^{\prime}$; consequently the range of $\theta$ in the latter case is greater than in the former by above half a degree; an excess, which, being nearly the 30 th part of the value of $\theta$ for $B$, and about the 20 th part of the whole range of $\theta$, must be expected to produce some manifest discrepancy of results.

As no applications of the formula (2.) have ever, to my knowledge, been made public, I have appended the following tables, which are computed by it. The second and third columns contain the indices of refraction by experiment and by theory, and the last shows the error of theory. In the first table $\mathrm{H}=1.6083$, and for line $\mathrm{B}, \theta=15^{\circ} 21^{\prime}{ }^{\prime}$; in the second table, the corresponding quantities are $\mathrm{H}=1 \cdot 5548$ and $\theta=22^{\circ} 43^{\prime}$.

\begin{tabular}{|c|c|c|c|c|c|c|c|}
\hline \multicolumn{4}{|c|}{ Flint Glass, No. 13.} & \multicolumn{4}{|c|}{ Oil of Cassia, No.i. } \\
\hline Ray. & True Index. & Computed. & Error. & Ray. & True Index. & Computed. & Error. \\
\hline B & $1 \cdot 6277$ & & & B & $1 \cdot 5963$ & & \\
\hline C & $1 \cdot 6297$ & $1 \cdot 6297$ & 0 & $\mathrm{C}$ & 1.6007 & $1 \cdot 6009$ & +2 \\
\hline D & $1 \cdot 6350$ & $1 \cdot 6347$ & -3 & D & $1 \cdot 6104$ & $1 \cdot 6131$ & +27 \\
\hline E & 1.6420 & 1.6422 & +2 & $\mathrm{E}$ & 1.6249 & 1.6297 & +48 \\
\hline $\mathbf{F}$ & $1 \cdot 6483$ & $1 \cdot 6486$ & +3 & F & $1 \cdot 6389$ & $1 \cdot 6449$ & $\begin{array}{r}60 \\
+60\end{array}$ \\
\hline $\mathrm{G}$ & $1 \cdot 6603$ & $1 \cdot 6609$ & +6 & G & $1 \cdot 6698$ & $1 \cdot 6723$ & +25 \\
\hline $\mathrm{H}$ & $1 \cdot 6711$ & & & H & $1 \cdot 7039$ & & \\
\hline
\end{tabular}

The constants $\mathrm{H} \mathrm{A}$ were in both these cases determined by assuming the indices for $\mathrm{B}$ and $\mathrm{H}$ to be the same by theory as experiment. 
How much soever we might be disposed, after inspecting the former of these tables, to think it confirmatory of theory, the wide discrepancies of the latter entirely obliterate the favourable impression. If upon the same line of abscissæe we construct two curves, the ordinates of which respectively represent the values of $\mu$ as found by theory and by experiment, the abscissa being proportional to the corresponding values of $\frac{1}{\lambda}$, we find that for flint glass they intersect in four points, the first and last of which are the extreme points, which were assumed to be the same in the two curves; the other two points are at $\mathrm{C}$, and a point about midway between $\mathrm{D}$ and $\mathrm{E}$. $\mathrm{An}$ inspection of the figure will show, that for about a third part of the whole there is a kind of proximity of which we might be disposed to think favourably, were it not that in the remaining $\frac{2}{3}$ rds of the figure there is precisely the same sort of dissimilarity as is exhibited in a magnified form by the oil of cassia, where there is no temptation to consider the curves as having the least trace of similarity, for they have merely common extremities. To exhibit this in the case of oil of cassia in a still stronger light, $I$ have determined the constants $H$ and $A$, on the supposition that the two curves coincide for letters $B$ and $F$. The following table exhibits the errors for the other fixed lines :-

\begin{tabular}{|c|c|c|c|c|c|c|}
\hline B & C & D & E & F & G & H \\
\hline$*$ & -5 & +6 & +7 & $*$ & -34 & -149 \\
\hline
\end{tabular}

From this statement it will be clear that the errors of theory in the case of the oil of cassia are far too great to allow of their being ascribed to the experimental results; and when we consider the extreme accuracy of Fraunhofer, by whom the data for the flint glass are furnished, and that it has just been shown that the errors in this case are of the same nature, though not so great, as in the other, I am inclined to assert that they are also as real in one case as in the other, and am led to infer, that the formula dedaced from M. Cauchy's theory does not agree sufficiently with experiment to warrant us in considering "the opprobrium of all theories of light" as having been completely removed by it from the undulatory theory.

As, however, the formula we have been considering is the one uniformly arrived at by all who have written on the subject, whatever were the peculiar hypotheses upon which their 
investigations were commenced and conducted, we ought not hastily to throw it aside. It has besides a strong claim upon our indulgence arising from the fact stated by Prof. Powell, "that it is certain that such a formula affords the closest accordance with truth throughout the whole range of low-dispersive substances; and even among many of the higher it gives a very near approach to such an agreement." The following remark may perbaps on this account be worthy of notice.

The constant represented by $A$ in equation (1.) is equal to $\pi \Delta x$, where $\Delta x$ is the distance between two adjacent particles of the refracting medium. Now in flint glass for the ray B Professor Powell found $\frac{\pi \Delta x}{\lambda}=16^{\circ} 10^{\prime}$; from which it follows that $\frac{\lambda}{\Delta x}$ is less than 12 ; for the ray $H$ it is less than $7:$ and in oil of cassia it is less than $5 . \frac{\lambda}{\Delta x}$ is the number of particles of the medium which lie within a wave's length; and it has always appeared to me highly improbable that this number should ever be so small as 5,7 , or 12; in fact it seems more likely (from the analogy of sound) that it ought to be a very high number. On this ground I feel less reluctance in yielding to the force of the argument before stated. It is also worth inquiring what effect the comparative largeness of $\Delta x$ (if allowed) may produce upon the convergency of the series which occur in the investigations from which our formula for $\mu$ is derived.

This letter having already exceeded the limit which I proposed to myself in setting out, it is necessary that 1 should state in as few words as possible my remarks on the other methods which have been made use of in the verification of theory. The principle of them amounts to this:-A series in inverse powers of $\lambda$ with indeterminate coefficients is assumed to represent $\mu$. This series is then assumed to be so rapidly convergent that all the terms after the first three may be omitted : the coefficients of these terms are found by assuming that the abridged series accurately gives the values of $\mu$ for three of the seven fixed lines; and lastly, the remaining four lines are used as a test of the truth of the undulatory theory. Now I cannot refrain from asking, what bas all this process of assumption to do with the undulatory theory? Surely it will not be denied, that it is a common and long-used method of interpolation. Then where has such a connexion been proved to exist between the undulatory theory and the principles of interpolation, as to make it follow, that when the latter 
furnishes true results the former is necessarily true? Perhaps it will be said that the undulatory theory suggested the form of the assumed series. It is a sufficient answer to this, that that form might have been conjectured from the mere inspection of the corresponding values of $\lambda$ and $\mu$. After all, are we sure that the form suggested is the correct one? Let any one, who is inclined to think that it is, determine the coefficients from the lines B, C, D, and with them calculate the indices for $\mathrm{E}, \mathrm{F}, \mathrm{G}, \mathrm{H}$, and I entertain no doubt he will be soon willing to resign the opinion. Again, if the method be one of interpolation only, it will naturally follow that the more widely the quantities are separated between which we interpolate, ccleris paribus, the greater are the errors to be expected in our results. Now this is so invariably found to be the case, that I believe Professor Powell has allowed that the errors increase with the greater dispersion of the substances employed. But that which strongly inclines me to the opinion that the method we are discussing is wholly unconnected with the truth of the undulatory theory, or indeed of any theory, and is a method of interpolation only, is the fact, that in the first applications of the method the same inadvertence with regard to the values of $\lambda$ was committed as $I$ have before alluded to, as occurring in "Researches Nos. I. II. III.," yet the results, though deduced from values of $\lambda$ not countenanced by theory, were hailed as confirmatory of the truth of theory.

But it is time to conclude these remarks, and therefore I will only add, as a general observation, that as the index for letter $\mathrm{G}$ is notoriously the most refractory, no method is in my opinion worth attention, even though it were furnished by theory, in which it is necessary to assume the indices for both $\mathrm{F}$ and $\mathrm{H}$ in order "to tame that ray." Yet even with the assumption of these, the tables for the more highly dispersive substances given in Professor Powell's book exhibit errors which show that $G$ is as untamed as ever, to say nothing of the other lines in several instances. On these grounds, I cannot avoid coming to the conclusion, that the methods of computation employed in compiling the tables contained in the book just referred to are such as are wholly unconnected with a physical theory of dispersion, and therefore their results, were they even coincident with experiment, add nothing to the strength of M. Cauchy's theory; and, were they even more discordant than they are with experiment, tend in no degree to overturn it. In this opinion I may perhaps be singular; but as it is just possible that a similar idea may have presented itself to others who, like myself, wish to have the impression, if erroneous, removed, I have ventured to make 
my doubts public, and shall be thankful if by doing so I should be so fortunate as to elicit an explanation from some of your more experienced correspondents.

I am, Sir, your obedient Servant,

January 1, 1842.

S. Earnshaw.

XLVI. On Atmospheric Refraction. By the Rev. R. MuRPHY, M.A., \&c.*

\section{§1. Hypothesis.}

THE refracting power of the atmosphere is a function of its distance from the centre of the earth, and tends in the direction of that centre. The curve described by a luminous ray in its passage through the air is governed by the usual laws of the trajectories of bodies acted on by centripetal forces.

$\$ 2$.- Let the velocity of light entering the atmosphere $=1$, and $v$ that at which it arrives at the earth's surface.

Let $r$ be the distance of a point in the trajectory of the ray from the earth's centre, and $\phi(r)$ the force acting then on light; we have

$$
v^{2}=1+2 f_{r} \phi(r)
$$

the limits of $r$ are 1 (the earth's radius) and $1+h$ ( $h$ being the height of the atmosphere). Hence $v^{2}$ is constant for all incidences, and we may put $v=1+m$ where $m$ is a certain constant.

$\S 3 .-$ Let $\theta$ be the angle made by the radius vector drawn to the earth's centre when the ray enters the atmosphere with that ray, and $z^{\prime}$ be the apparent zenith distance.

The perpendicular on the ray as it enters the atmosphere drawn from the earth's centre $=(1+h) \sin \theta$, and that as it enters the eye $=\sin z^{\prime}$. These are inversely proportional to the corresponding velocities, viz. 1 and $1+m$; hence

$$
(1+h) \cdot \sin \theta=(1+m) \sin z^{\prime} \text {. }
$$

$\S 4$.- Let the observed body be the moon, at a distance $a$, reckoning in radii of the earth, from the centre.

Let $z$ be its true zenith distance seen from the earth's centre, corresponding to the apparent zenith distance $z^{\prime}$.

Let $p$ be the moon's parallax at that zenith distance, and let $\omega$ be the angle at the moon subtended by the bent trajectory of the luminous ray. Then $p+\omega$ is the angle at the moon made by the radius vector from the earth's centre with the issuing ray, which being the same that enters the atmosphere to reach the eye, we have

$$
a \sin (p+\omega)=(1+h) \cdot \sin \theta \text {. }
$$

* Communicated by the Author. 